\title{
Circulating microparticles from patients with valvular heart disease and cardiac surgery inhibit endothelium-dependent vasodilation
}

\author{
Li Fu, MD, ${ }^{\text {a,b,c }}$ Xiao-Xia Hu, MD, ${ }^{\text {b,c }}$ Ze-Bang Lin, MD, ${ }^{\text {b,c }}$ Feng-Jun Chang, MD, PhD, ,b,c \\ Zhi-Jun Ou, MD, ${ }^{\mathrm{b}, \mathrm{c}, \mathrm{d}}$ Zhi-Ping Wang, MD, PhD, ${ }^{\mathrm{a}, \mathrm{b}}$ and Jing-Song Ou, MD, $\mathrm{PhD}^{\mathrm{a}, \mathrm{b}, \mathrm{c}}$
}

\begin{abstract}
Objective: Vascular function is very important for maintaining circulation after cardiac surgery. Circulating microparticles (MPs) generated in various diseases play important roles in causing inflammation, coagulation, and vascular injury. However, the impact of MPs generated from patients who have valvular heart disease (VHD), before and after cardiac surgery, on vascular function remains unknown. This study is designed to investigate the impact of such MPs on vasodilation.
\end{abstract}

Methods: Microparticles were isolated from age-matched healthy subjects and patients who had VHD, before cardiac surgery, and at 12 hours and 72 hours afterward. The number of MPs was measured and compared. Effects evaluated were of the impact of MPs on: vasodilation of mice aorta; the phosphorylation and expression of Akt, endothelial nitric oxide synthase (eNOS), protein kinase C- $\beta$ II (PKC- $\beta$ II), and p70 ribosomal protein S6 kinase (p70S6K); expression of caveolin-1; the association of eNOS with heat shock protein 90 (HSP90); and generation of nitric oxide and superoxide anion of human umbilical vein endothelial cells.

Results: Compared with the healthy subjects, VHD patients had significantly higher levels of circulating MPs and those MPs before cardiac surgery can: impair endothelium-dependent vasodilation; inhibit phosphorylation of Akt and eNOS; increase activation of PKC- $\beta$ II and p70S6K; enhance expression of caveolin-1; reduce the association of HSP90 with eNOS; decrease nitric oxide production, and increase superoxide anion generation. These deleterious effects were even stronger in postoperative MPs.

Conclusions: Our data demonstrate that MPs generated from VHD patients before and after cardiac surgery contributed to endothelial dysfunction, by uncoupling and inhibiting eNOS. Circulating MPs are potential therapeutic targets for the maintenance of vascular function postoperatively. ( $\mathrm{J}$ Thorac Cardiovasc Surg 2015;150:666-72)

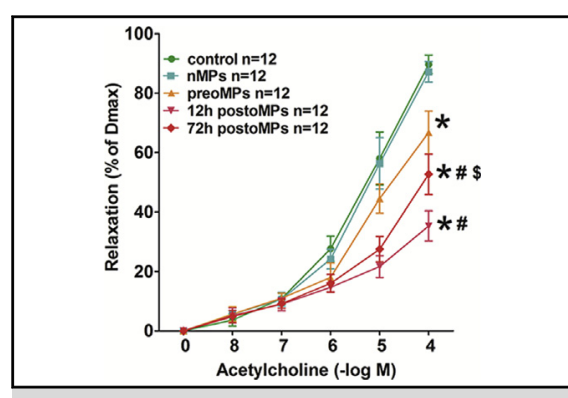

Microparticles from patients who had VHD and underwent cardiac surgery were found to impair endothelium-dependent vasodilation.

\section{Central Message}

Microparticles from VHD patients before and after cardiac surgery were found to impair endothelial function, and therefore, may be appropriate therapeutic targets.

\section{Perspective}

Microparticles play important roles in vascular injury. We demonstrated that MPs from VHD patients before and after cardiac surgery can impair endothelium-dependent vasodilation by uncoupling eNOS and inhibiting its activity. Our findings can partly explain the hemodynamic instability that occurs after cardiac surgery, indicating that MPs are potential therapeutic targets for increasing hemodynamic stability.

See Editorial Commentary page 673.
From the ${ }^{\mathrm{a} D i v i s i o n}$ of Cardiac Surgery, ${ }^{\mathrm{b}}$ Key Laboratory of Assisted Circulation, Ministry of Health, ${ }^{\mathrm{c}}$ Guangdong Province Engineering Laboratory for Diagnosis and Treatment of Vascular Diseases, and ${ }^{\mathrm{d}}$ Division of Hypertension and Vascular Diseases, The First Affiliated Hospital, Sun Yat-sen University, Guangzhou, People's Republic of China.

This study was supported by the National Natural Science Foundation of China (81170271, 81370370, and Distinguished Young Scholar 81325001), the Changjiang Scholars Program from the Ministry of Education of China, the Guangdong Pearl River Scholars Program, the 973 project (2009CB522104), and the International Cooperation Project (2015DFA31070) from the Ministry of Science and Technology of China, Sun Yat-Sen University Clinical Research 5010 Program; the Ministry of Education of China (20100171110057), the Program of National Key Clinical Specialties, Science and Technology Research for the Returned Over- seas Chinese Scholars from the Ministry of Human Resources and Social Security of China (2011).

L. Fu and X.-X. Hu contributed equally to this study.

Received for publication Feb 12, 2015; revisions received May 19, 2015; accepted for publication May 23, 2015; available ahead of print July 2, 2015.

Address for reprints: Jing-Song Ou, MD, PhD, Division of Cardiac Surgery, The First Affiliated Hospital, Sun Yat-sen University, 58 Zhong Shan Er Rd, Guangzhou 510080, People's Republic of China (E-mail: oujs@mail.sysu.edu.cn or oujs2000@yahoo.com).

$0022-5223 / \$ 36.00$

Copyright (c) 2015 by The American Association for Thoracic Surgery

http://dx.doi.org/10.1016/j.jtcvs.2015.05.069 


\section{Abbreviations and Acronyms \\ eNOS = endothelial nitric oxide synthase \\ HUVEC = human umbilical vein endothelial cell \\ L-NAME $=$ NG-nitro-L-arginine methyl ester \\ $\mathrm{MP}=$ microparticle \\ $\mathrm{PKC}-\beta \mathrm{II}=$ protein kinase $\mathrm{C}-\beta \mathrm{II}$ \\ p70S6K $=$ p70 ribosomal protein S6 kinase \\ Ser $=$ serine \\ VHD $=$ valvular heart disease}

Circulating microparticles (MPs) are a group of membrane vesicles that are released by cell activation or apoptosis. Studies have demonstrated that circulating MPs, or certain subgroups of MPs, increase in various cardiovascular diseases, such as hypertension, acute coronary syndromes, and mitral valve disease. ${ }^{1-3}$ In addition, MPs have important physiologic and pathophysiologic functions, including coagulation, inflammation, and vascular function. ${ }^{4,5}$

Valvular heart disease (VHD) is among the main heart problems globally. Most VHD patients need cardiac surgery to repair or replace the defective valve under cardiopulmonary bypass. During the perioperative period of such surgery, hemodynamic status is unstable. In addition, such surgery can lead to a systemic inflammatory response and cause difficulties in maintaining circulation stability. ${ }^{6}$ Preventza and colleagues ${ }^{7}$ additionally reported that the time to complete cardiopulmonary bypass was related to mortality after surgery.

Recently, we demonstrated that vascular function was impaired by proinflammatory high-density lipoprotein during cardiac surgery in VHD patients, via uncoupling of endothelial nitric oxide synthase (eNOS) ${ }^{8}$ Boulanger and colleagues 9 showed that MPs from patients with myocardial infarction impaired endothelium-dependent vasodilation. Martin and colleagues 5 further demonstrated that endothelial function can be altered by $\mathrm{T}$ lymphocyte-derived MPs, through effects on nitric oxide and regulation of eNOS and caveolin-1. Therefore, in the present study, we investigated whether MPs from VHD patients undergoing cardiac surgery would lead to vascular dysfunction.

\section{METHODS}

\section{Study Populations}

A total of 42 patients diagnosed with VHD were recruited from the First Affiliated Hospital, Sun Yat-sen University. Patients who suffered from coronary heart disease, infectious disease, diabetes, renal failure, or severe trauma, or who had undergone surgery within the preceding 3 months, were excluded. All studied subjects were age $>18$ years. A total of 22 healthy subjects age $>18$ years were enrolled as a control group. This study was approved by the Ethics Review Board of the First Affiliated Hospital, Sun Yat-sen University. Informed consent was obtained from all subjects enrolled in this study. The clinical characteristics of the study population are described in Table 1 and operation data and postoperative drug of the VHD patients are described in Table 2.

\section{Isolation of Blood Samples}

Blood samples were drawn from the healthy subjects, and from the patients both before, and at 12 and 72 hours after, they underwent cardiac surgery with cardiopulmonary bypass. MPs were isolated as described elsewhere. ${ }^{9}$ Briefly, the blood samples were centrifuged at $11,000 \mathrm{~g}$ for 2 minutes, at room temperature, to obtain platelet-poor plasma. About $50 \mu \mathrm{l}$ of this plasma was immediately frozen and stored at $-80^{\circ} \mathrm{C}$ for flow cytometry analysis. The rest was centrifuged at $13,000 \mathrm{~g}$ for 45 minutes, to yield MPs, which were resuspended in RPMI (Roswell Park Memorial Institute) medium and then frozen and stored at $-80^{\circ} \mathrm{C}$.

\section{Flow Cytometry Analysis}

The platelet-poor plasma was thawed on ice, and $10 \mu \mathrm{l}$ was incubated with annexin $\mathrm{V}$ that had been previously diluted in $90 \mu \mathrm{l}$ of annexin $\mathrm{V}$ binding buffer ( $2 \mathrm{X}$, containing $20 \mathrm{mM}$ HEPES (N-2-hydroxyethylpiperazine$\mathrm{N}$-2-ethane sulfonic acid), $0.28 \mathrm{M}$ sodium chloride, and $5 \mathrm{mM}$ calcium chloride; $\mathrm{pH} 7.5$ ), for 30 minutes, at room temperature, in the dark. Platelet-poor plasma incubated with annexin $\mathrm{V}$ in $2 \mathrm{X}$ binding buffer, containing 5 mM EDTA (ethylenediaminetetraacetic acid) but no calcium chloride, was used as a negative control. Before analysis, $10 \mu \mathrm{l}$ of flow count calibrator beads (Beckman Coulter Inc, Fullerton, Calif) with known concentration were added into the samples. The annexin $\mathrm{V}$ binding MPs were analyzed in MoFlo XDP (Beckman Coulter Inc). After exclusion of background noise, those positively labeled for annexin $\mathrm{V}$ in the gate ( size $=1 \mu \mathrm{m}$ ) were considered to be MPs.

\section{Vasodilation Study}

The experimental protocol was approved by the Animal Ethics Commission of the First Affiliated Hospital, Sun Yat-sen University. All mice used were purchased from the Experimental Animal Center of Sun Yat-sen University. The vasodilation study was performed as described elsewhere. $^{8}$

Briefly, male C57BL/6 (C57 black 6) mice were killed with pentobarbital (Sigma-Aldrich, St Louis, Mo), and aortas were extracted. Aortic rings, $3 \mathrm{~mm}$ wide, were obtained and connected to an isometric force transducer (DMT620M, ADInstruments, New South Wales, Australia). Aortic rings were suspended in organ chambers filled with Krebs solution and put through a 30-minute equilibration period with no tension. After that, the pretension was gradually increased to $5 \mathrm{mN}$, followed by resting for 60 minutes. Next, the rings were exposed to $60-\mathrm{mmol} / \mathrm{L}$ potassium chloride and washed out at least 3 times to achieve optimal tension. The aortic rings were treated with $4.6 \times 10^{6}$ per $\mathrm{mL}$ of MPs (the pathologic concentration of MPs in VHD patients before cardiac surgery) for 30 minutes and then preconstricted with $10^{-6} \mathrm{~mol} / \mathrm{L}$ noradrenaline (Sigma-Aldrich). Endothelium-dependent vasodilation was determined by acetylcholine $\left(10^{-8}\right.$ to $\left.10^{-4} \mathrm{~mol} / \mathrm{L}\right)$ and NG-nitro-L-arginine methyl ester (L-NAME, $100 \mu \mathrm{mol} / \mathrm{L}$; both from Sigma-Aldrich), respectively. Endothelium-independent vasodilation was studied using the nitrovasodilator sodium nitroprusside $\left(10^{-8}\right.$ to $10^{-4} \mathrm{~mol} / \mathrm{L}$; SigmaAldrich). Aortic rings with no pretreatment were used as controls.

\section{Western Blot Analysis}

Passage-4 human umbilical vein endothelial cells (HUVECs) were cultured in an endothelial cell medium (both from ScienCell Research Laboratories, Carlsbad, Calif) supplemented with 5\% fetal bovine serum, $1 \%$ growth factors, and $1 \%$ penicillin/streptomycin. After serum starvation with $0.5 \%$ fetal bovine serum for 24 hours, HUVECs were treated with MPs $\left(4.6 \times 10^{6}\right.$ per $\left.\mathrm{mL}\right)$ for 1 hour. Cellular proteins were obtained, and western blotting was performed as described elsewhere. ${ }^{10}$ Passage- 4 HUVECs treated with phosphate-buffered saline were used as controls.

Antibodies of Akt, phosphorylation of Akt, phosphorylation of eNOS at Ser1177 (serine 1177 site), and caveolin-1 were from Cell Signaling Technology Inc (Danvers, Mass). Antibody of eNOS (sc-654), 
TABLE 1. Clinical characteristics

\begin{tabular}{lcc}
\hline \multicolumn{1}{c}{ Variables } & $\begin{array}{c}\text { Control group } \\
(\mathbf{n}=\mathbf{2 2})\end{array}$ & $\begin{array}{c}\text { VHD patients } \\
(\mathbf{n}=\mathbf{4 2})\end{array}$ \\
\hline Age $(\mathrm{y})$ & $47.1 \pm 10.2$ & $48.0 \pm 9.8$ \\
Gender, male (n) & $12 / 22$ & $20 / 42$ \\
NYHA class (II/III/IV) & $0 / 0 / 0$ & $11 / 28 / 3$ \\
Left atrial diameter $(\mathrm{mm})$ & $30.8 \pm 2.2$ & $46.8 \pm 9.0^{*}$ \\
LVEDD $(\mathrm{mm})$ & $43.8 \pm 2.6$ & $55.5 \pm 8.8^{*}$ \\
LVESD $(\mathrm{mm})$ & $29.8 \pm 2.7$ & $37.9 \pm 7.6^{*}$ \\
LV ejection fraction $(\%)$ & $69.3 \pm 8.7$ & $59.3 \pm 8.5^{*}$ \\
\hline
\end{tabular}

Values are expressed as mean \pm SD. $V H D$, Valvular heart disease; NYHA, New York Heart Association; $L V E D D$, left ventricular end-diastolic dimension; $L V E S D$, left ventricular end-systolic dimension; $L V$, left ventricular. $* P<.05$.

protein kinase C- $\beta \mathrm{II}$ (PKC- $\beta \mathrm{II})$, p70 ribosomal protein S6 kinase (p70S6K), phosphorylation of PKC- $\beta$ II at Ser660, and p70S6K at Ser411, were from Santa Cruz Biotechnology (Santa Cruz, Calif). Glyceraldehyde 3-phosphate dehydrogenase (GAPDH) antibody was from Proteintech Group (Chicago, Ill).

\section{Immunoprecipitation}

The HUVECs were starved with $0.5 \%$ fetal bovine serum for 24 hours, followed by treatment with MPs $\left(4.6 \times 10^{6}\right.$ per $\left.\mathrm{mL}\right)$ for 1 hour. Next, cells were lysed in radioimmunoprecipitation assay buffer, and samples were collected as described elsewhere. ${ }^{3}$ Cell lysates were incubated with anti-eNOS antibody (sc-136977; Santa Cruz Biotechnology) overnight, for immunoprecipitation of eNOS. The samples were mixed with Laemmli buffer and heated $\left(95^{\circ} \mathrm{C}, 5\right.$ minutes). Two minutes after being cooled on ice, the mixtures were centrifuged, and the supernatants were immunoblotted for eNOS and heat shock protein 90 (Santa Cruz Biotechnology). Passage-4 HUVECS treated with phosphate buffered saline were used as controls.

TABLE 2. Operation data and postoperative drugs

\begin{tabular}{lc}
\hline \multicolumn{1}{c}{ Variables } & VHD patients $(\mathbf{n}=\mathbf{4 2})$ \\
\hline CPB time (min) & $137.3 \pm 48.2$ \\
Clamping time (min) & $90.1 \pm 33.1$ \\
Operation performed & \\
MVR & $3 / 42$ \\
MVP & $1 / 42$ \\
AVR & $5 / 42$ \\
TVR & $1 / 42$ \\
MVR+AVR & $6 / 42$ \\
MVR+TVP & $16 / 42$ \\
MVR+TVR & $2 / 42$ \\
AVR+TVP & $1 / 42$ \\
MVR+AVR+TVP & $6 / 42$ \\
MVR+AVR+TVR & $1 / 42$ \\
Postoperative medications & \\
Dopamine & $42 / 42$ \\
Dobutamine & $36 / 42$ \\
Norepinephrine & $21 / 42$ \\
Epinephrine & $15 / 42$ \\
Isoproterenol & $20 / 42$ \\
Metaraminol & $26 / 42$ \\
Digitalis & $40 / 42$ \\
Milrinone & $21 / 42$ \\
Corticoids & $42 / 42$ \\
\hline$C P B$ Cadinlmar & \\
\hline
\end{tabular}

$C P B$, Cardiopulmonary bypass; $M V R$, mitral valve replacement; $M V P$, mitral valvuloplasty; $A V R$, aortic valve replacement; $T V R$, tricuspid valve replacement; $T V P$, tricuspid valvuloplasty.

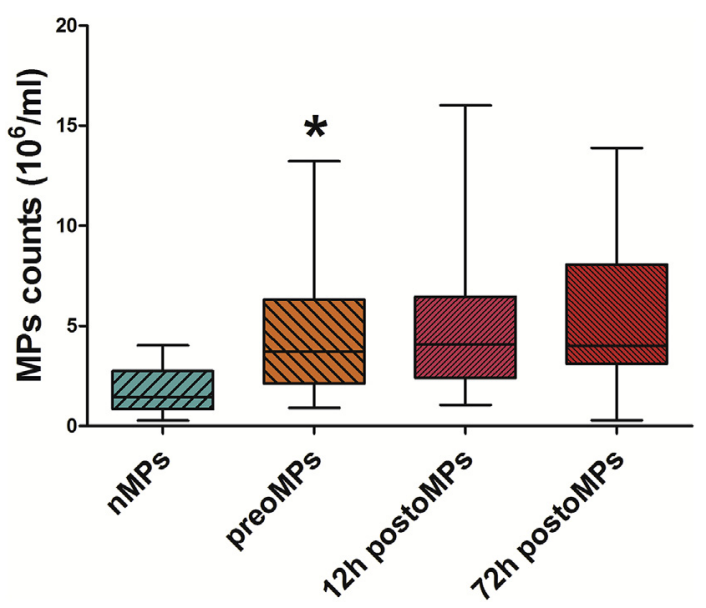

FIGURE 1. MPs were increased in VHD patients. Compared with nMPs, the amount of MPs was significantly higher in VHD patients before surgery. No significant difference was found among preoMPs, $12 \mathrm{~h}$ postoMPs, and $72 \mathrm{~h}$ postoMPs. $M P$, Microparticle; $n M P$, MPs from healthy subjects; preoMPs, MPs from VHD patients before surgery; $12 h$ postoMPs, MPs from VHD patients at 12 hours after cardiac surgery; $72 \mathrm{~h}$ postoMPs, MPs from VHD patients at 72 hours after cardiac surgery. $* P<.05$.

\section{Measurement of Nitric Oxide Generation}

Passage-4 HUVECs were cultured in 12-well plates and serum starved with $0.5 \%$ fetal bovine serum for 24 hours. All plates were incubated at $37^{\circ} \mathrm{C}$ for 1 hour in HBSS (Hank's balanced salt solution) containing $25 \mu \mathrm{mol} / \mathrm{L} \mathrm{L}$-arginine and MPs $\left(4.6 \times 10^{6}\right.$ per $\left.\mathrm{mL}\right)$. The supernatant was collected, and nitric oxide generation was measured by Griess assay (Nanjing Jiancheng Bioengineering Institute, Nanjing, China). Cellular proteins were collected for concentration determination by bicinchoninic acid protein assay (Merck, Kenilworth, NJ), as described elsewhere. ${ }^{10}$ Passage- 4 HUVECs treated with phosphate buffered saline were used as controls.

\section{Measurement of Superoxide Anion Generation}

The effects of MPs on superoxide anion production were assessed with the lucigenin $(5 \mu \mathrm{mol} / \mathrm{L})$ assay. ${ }^{11}$ Briefly, HUVECs were cultured in 48-well plates. After serum starvation with $0.5 \%$ fetal bovine serum for 24 hours, L-NAME $(1 \mathrm{mmol} / \mathrm{L})$ was added to one half of a plate and cultured for 30 minutes. MPs $\left(4.6 \times 10^{6}\right.$ per $\left.\mathrm{mL}\right)$ were added into HUVECs with and without pretreatment with L-NAME, and incubated at $37^{\circ} \mathrm{C}$ for another 30 minutes. Luminescence was measured with a SpectraMax M5/M5e multidetection reader (Molecular Devices, Sunnyvale, Calif), to detect superoxide anion production, and cellular proteins were harvested for determining protein concentrations via the bicinchoninic acid protein assay (Merck), as described elsewhere. ${ }^{11}$ Passage-4 HUVECs treated with phosphate buffered saline were used as controls.

\section{Statistical Analysis}

Statistical analyses were performed using SPSS 21.0 software (SPSS Inc, Chicago, Ill). For comparison of patients with healthy subjects, and between the impact of MPs with, versus without, L-NAME on superoxide anion production, an independent 2- sample $t$ test was used. For comparison of control, healthy subjects, and patients, a 1-way ANOVA and the Newman-Keuls test were used. For comparing before-surgery and aftersurgery (at various time points) measures, a repeated measures analysis with Bonferroni correction was applied. A $\chi^{2}$ analysis was used to compare proportions between different groups. Data are presented as mean $\pm \mathrm{SD}$. 

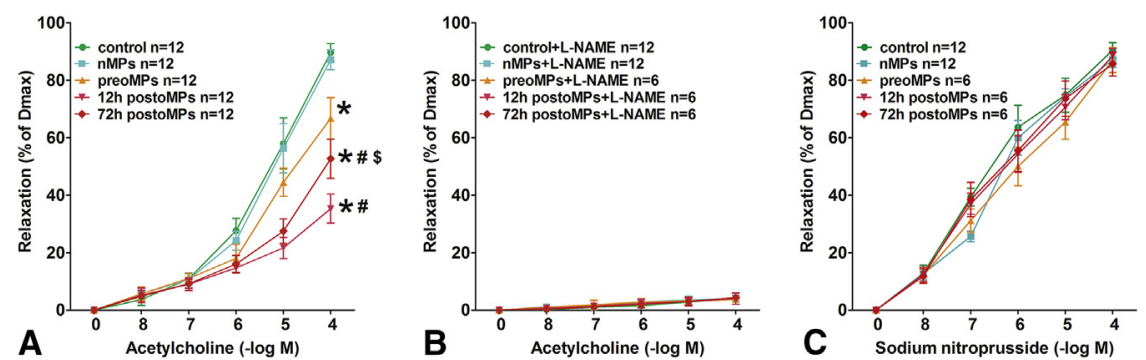

FIGURE 2. Microparticles from VHD patients before and after cardiac surgery impaired endothelium-dependent vasodilation. Because the maximum relaxation (Dmax \%) induced by acetylcholine and sodium nitroprusside was shown at $10^{-4} \mathrm{~mol} / \mathrm{L}$, the relaxation of this point was calculated and compared. A, No difference was found in vasodilation between control MPs and nMPs. Compared with nMPs, preoMPs significantly inhibited acetylcholine-induced vasodilation. The impaired vasodilation by postoMPs was more prominent, especially for $12 \mathrm{~h}$ postoMPs. B, After treatment with L-NAME, acetylcholineinduced vasodilation was completely inhibited in each group. C, No difference was found in sodium nitroprusside-induced vasodilation among control MPs, nMPs, and preoMPs, or among preoMPs, $12 \mathrm{~h}$ postoMPs, and $72 \mathrm{~h}$ postoMPs. DMax $\%$, Maximum relaxation; $n M P$, MPs from healthy subjects; preoMPs, MPs from VHD patients before surgery; $12 \mathrm{~h}$ postoMPs, MPs from VHD patients at 12 hours after cardiac surgery; $72 \mathrm{~h}$ postoMPs, MPs from VHD patients at 72 hours after cardiac surgery. *, Versus control and nMPs; \#, versus preoMPs; \$, versus $12 \mathrm{~h}$ postoMPs. $P<.05 ; \mathrm{n}=6-12$.

\section{RESULTS \\ Clinical Characteristics}

Clinical characteristics are shown in Table 1. No significant difference was found in age or gender of VHD patients compared with healthy subjects. No signs of morphologic heart disease were detected, using echocardiography, in the control group. Left atrial diameter, left ventricular end-diastolic dimension, and left ventricular end-systolic dimension were significantly larger in the patient group than in the control group. Left ventricular ejection fraction was lower in the patient group than in the control group. The operation data and postoperative drugs are shown in Table 2.

\section{The Levels and Effects of Circulating MPs}

Compared with healthy subjects, VHD patients had significantly higher levels of circulating MPs. No significant difference was found in MP numbers for VHD patients at different time points (Figure 1).

Compared with MPs from healthy subjects, MPs from VHD patients markedly decreased the endotheliumdependent relaxation in response to acetylcholine. Compared with MPs from VHD patients before surgery, MPs from after surgery, especially at 12 hours, impaired vasodilation to a greater extent (Figure 2, A). Acetylcholine-induced vasodilation was completely inhibited by L-NAME in various MP-treated groups (Figure 2, B). However, endothelium-independent vasodilation response to sodium nitroprusside was not affected by those MPs (Figure 2, $C$ ).

Microparticles from VHD patients, especially those from after surgery, significantly reduced phosphorylation of Akt and eNOS at Ser1177, without alteration of AKT and eNOS expression (Figure 3, $A$ and $B$ ). Compared with MPs from healthy subjects, MPs from VHD patients significantly enhanced the phosphorylation of PKC- $\beta$ II at Ser660, and
p70S6K at Ser411, without alteration of PKC- $\beta$ II and p70S6K expression (Figure 3, $C$ and $D$ ). Compared with MPs from healthy subjects, MPs from VHD patients, especially the postoperative ones, significantly increased the expression of caveolin-1 (Figure 3, E).

Microparticles from healthy subjects did not affect the association of heat shock protein 90 with eNOS, whereas MPs from VHD patients dramatically inhibited this association. This phenomenon was more obvious for the MPs from 12 hours and 72 hours after cardiac surgery.

The MPs from VHD patients, especially those obtained after cardiac surgery, significantly decreased nitric oxide production (Figure 4, A). The MPs from VHD patients before surgery significantly increased superoxide anion generation; and the MPs from VHD patients from 12 hours and 72 hours after surgery stimulated superoxide anion generation even more. Moreover, the increased generation of superoxide anion was inhibited by L-NAME (Figure 4, $B$ ).

\section{DISCUSSION}

To the best of our knowledge, this study is the first to focus on the function of MPs from VHD patients during the perioperative period of cardiac surgery. The data showed that MPs from VHD patients could decrease nitric oxide production and increase superoxide anion generation by uncoupling eNOS, thus impairing endothelium-dependent vasodilation. Cardiac surgery with cardiopulmonary bypass enhanced this effect. These findings indicate that MPs are potential therapeutic targets for the maintenance of vascular function and the prevention of poor outcomes for VHD patients.

Evidence has shown that circulating MPs increase in various diseases. ${ }^{12}$ Certain subgroups of MPs and circulating MPs in vivo can impair endothelial function. We and other researchers demonstrated that endothelial MPs can induce endothelial dysfunction by impairing nitric 

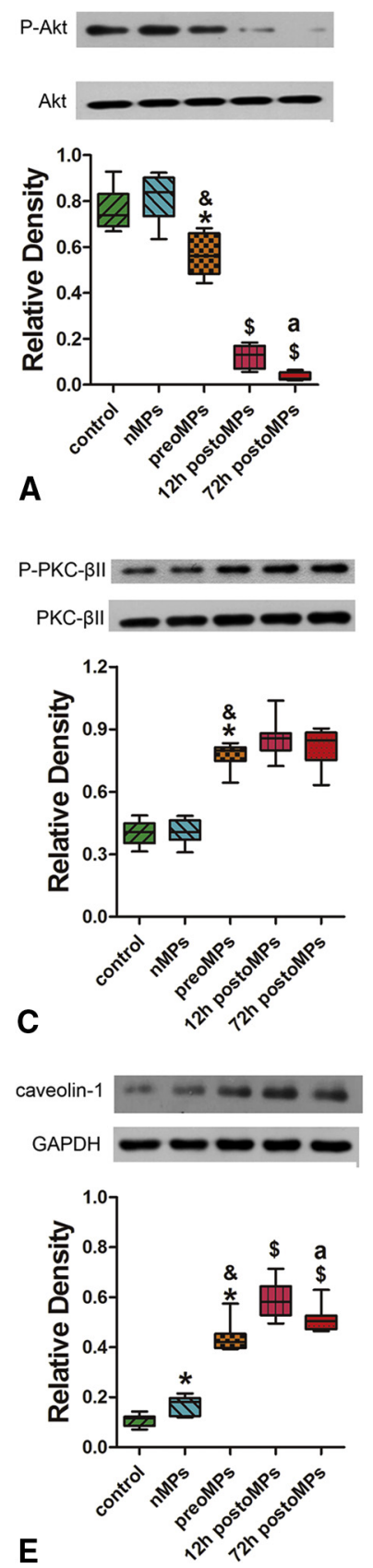
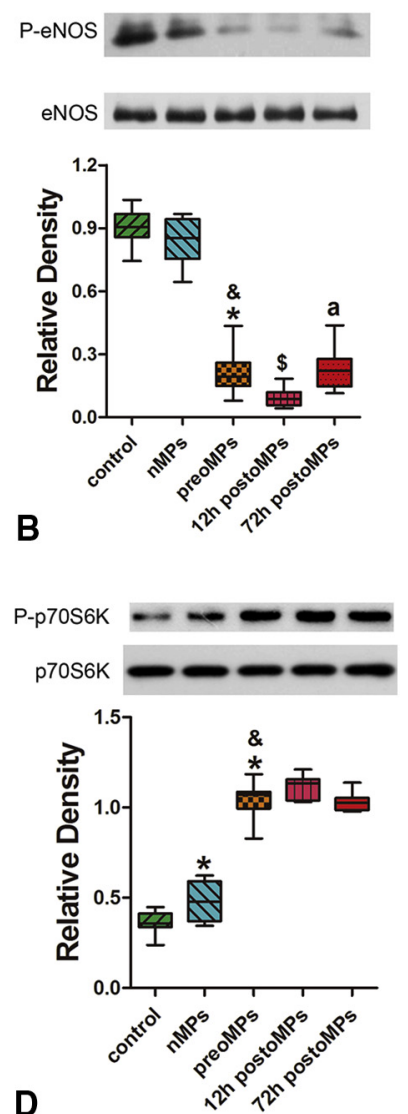

D
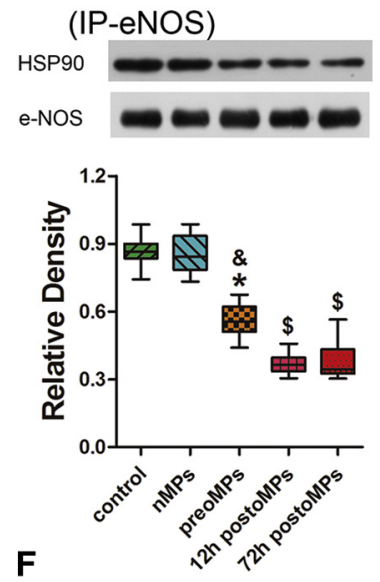

FIGURE 3. Microparticles from VHD patients inhibited eNOS signaling pathways. A and B, nMPs did not alter phosphorylation of Akt and eNOS at Ser1177, but MPs from VHD patients significantly inhibited phosphorylation of Akt, and of eNOS at Ser1177. The effects on phosphorylation of Akt were more prominent in the $12 \mathrm{~h}$ postoMPs and $72 \mathrm{~h}$ postoMPs, and the effects on phosphorylation of eNOS were more prominent in $12 \mathrm{~h}$ postoMPs. C and D, nMPs did not change phosphorylation of PKC- $\beta$ II, but increased phosphorylation of p70S6K. MPs from VHD patients significantly stimulated phosphorylation of PKC- $\beta$ II and p70S6K. No difference was found among preoMPs, $12 \mathrm{~h}$ postoMPs, and $72 \mathrm{~h}$ postoMPs. E, nMPs increased the expression of caveolin-1. But MPs from VHD patients increased caveolin-1 expression far more than did nMPs, which were seen even more in postoMPs. F, nMPs did not affect the association of HSP90 with eNOS; preoMPs significantly inhibited the association of

oxide bioavailability. ${ }^{13,14}$ Martin and colleagues ${ }^{5}$ reported that $\mathrm{T}$ cell-derived MPs reduced endothelial vasodilation and decreased expression of eNOS. In addition, in vivo MPs from patients who have acute myocardial infarction have been shown to impair endothelium-dependent vascular relaxation. ${ }^{9}$

Cardiac surgery with cardiopulmonary bypass is well recognized as being able to alter physiologic status, and induce systemic inflammatory reactions, by releasing interleukins, acute phase response, and functional change of certain blood properties. ${ }^{8,15-17}$ These abnormalities may impair vascular function and the stability of the hemodynamic system, which may contribute to the high morbidity and mortality seen during the perioperative period.

Circulating MPs have been reported to be high in patients undergoing such surgery. ${ }^{18}$ More important, van den Goor and colleagues ${ }^{19}$ reported that such MPs can be effectively removed by a cell saver device. Our data showed that, although the level of MPs in VHD patients was higher than that in healthy subjects, MP levels before versus after surgery were not significantly different. The MPs from VHD patients markedly impaired endothelium-dependent vasodilation. This effect was even stronger after cardiac surgery, and continued even 72 hours postoperatively. These data indicate, first, that MPs from VHD patients may contribute to the hemodynamic instability and physiologic abnormalities that occur after cardiac surgery. Second, they show that cardiac surgery with cardiopulmonary bypass may generate more-harmful MPs and result in reduced vascular functioning.

Intact endothelial-dependent vascular functioning is crucial for homeostasis, and eNOS-derived nitric oxide plays an important role in it. ${ }^{20}$ Reduced activation of eNOS is highly related to decreased nitric oxide bioavailability. In this study, we found that MPs from VHD patients, especially after cardiac surgery, can dramatically inhibit phosphorylation of Akt and phosphorylation of eNOS at the Ser1177 site, increase the expression of caveolin-1, and promote the phosphorylation of PKC- $\beta$ II and p70S6K. The phosphorylation of eNOS at the Ser1177

\footnotetext{
HSP90 with eNOS, and this was more pronounced for postoMPs. P-Akt, Phosphorylation of Akt; P-eNOS, phosphorylation of eNOS; $n M P$, MPs from healthy subjects; preoMPs, MPs from VHD patients before surgery; $12 \mathrm{~h}$ postoMPs, MPs from VHD patients at 12 hours after cardiac surgery; $72 h$ postoMPs, MPs from VHD patients at 72 hours after cardiac surgery; $P$-PKC- $\beta I I$, phosphorylation of PKC- $\beta \mathrm{II} ; P-p 70 \mathrm{~S} 6 \mathrm{~K}$, phosphorylation of p70S6K; $P K C-\beta I I$, protein kinase $\mathrm{C}-\beta \mathrm{II} ; p 70 \mathrm{~S} 6 \mathrm{~K}$, p70 ribosomal protein S6 kinase; IP-eNOS, immunoprecipitation of eNOS; $A k t$, protein kinase B; $H S P 90$, heat shock protein 90; GAPDH, glyceraldehyde 3-phosphate dehydrogenase; $V H D$, valvular heart disease; eNOS, endothelial nitric oxide synthase. *, Versus control; \&, versus nMPs; \$, versus preoMPs; a, versus $12 \mathrm{~h}$ postoMPs; $P<.05 ; \mathrm{n}=8$.
} 

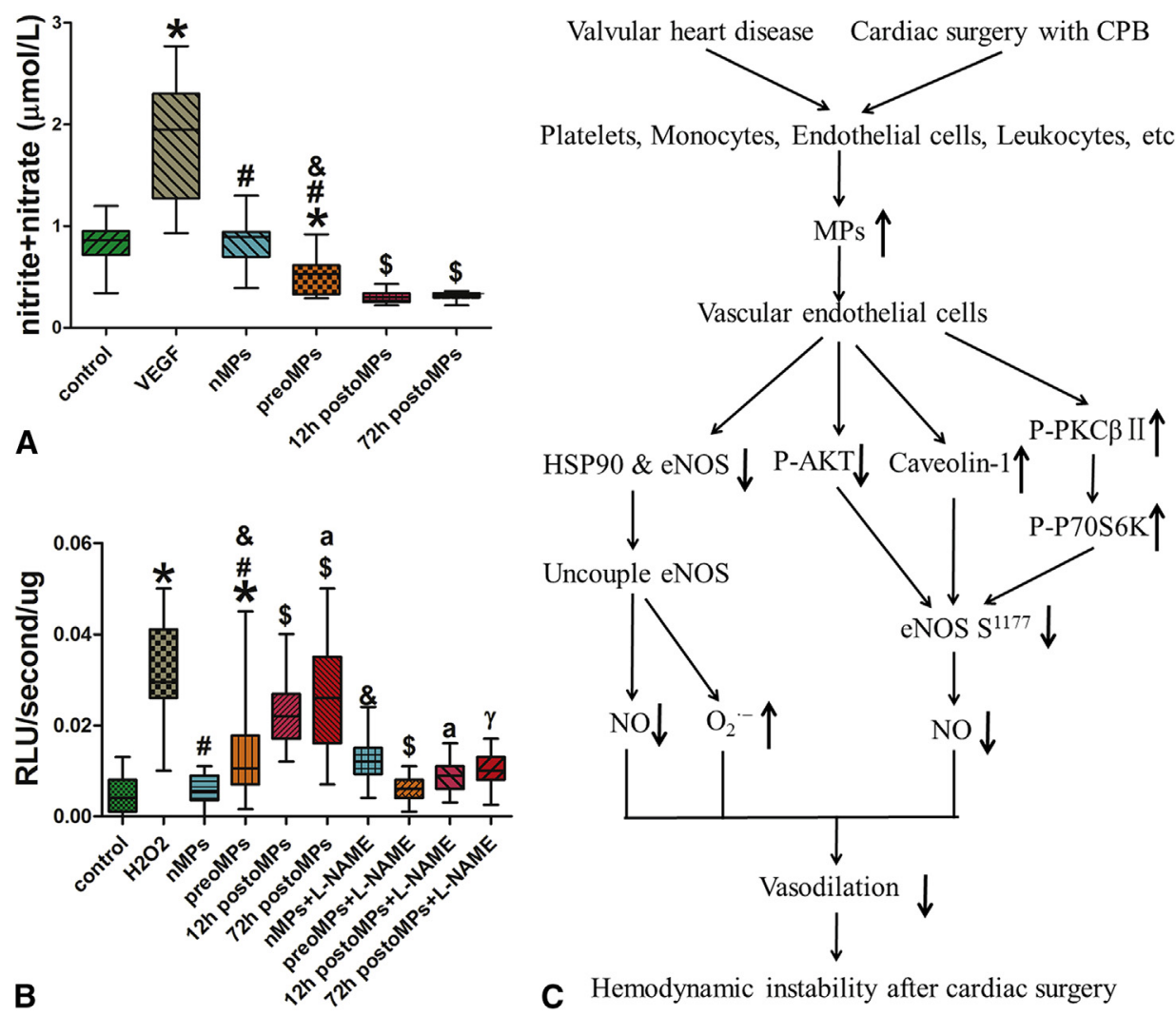

C Hemodynamic instability after cardiac surgery

FIGURE 4. MPs from VHD patients before and after cardiac surgery decreased nitric oxide production and increased generation of superoxide anion $\mathrm{O}_{2} \bullet-$, in the hypothetical working model. A, No difference was found in NO production between control MPs and nMPs. PreoMPs significantly decreased the production of NO, and postoMPs decreased it even more. *, Versus control; \#, versus VEGF; \&, versus nMPs; $\$$, versus preoMPs; $P<.05 ; \mathrm{n}=12$. B, No difference was found in $\mathrm{O}_{2} \bullet^{-}$generation between control MPs and nMPs. PreoMPs significantly increased $\mathrm{O}_{2} \bullet^{-}$generation; $12 \mathrm{~h}$ and $72 \mathrm{~h}$ postoMPs increased it even more, and could be inhibited by L-NAME. *, Versus control; \#, versus $\mathrm{H}_{2} \mathrm{O}_{2} ;$; , versus nMPs; \$, versus preoMPs; a, versus $12 \mathrm{~h}$ postoMPs; $\gamma$, versus $72 \mathrm{~h}$ postoMPs; $P<.05 ; \mathrm{n}=12$. C, Hypothetical working model: how MPs from VHD patients and cardiac surgery impair vasodilation and influence hemodynamic stability. The MP level was increased in VHD patients before and after cardiac surgery with CPB and these MPs could decrease eNOS activity by: inhibiting the Akt/eNOS pathway; enhancing the PKC- $\beta \mathrm{II} / \mathrm{p} 70 \mathrm{~S} 6 \mathrm{~K}$ pathway; and increasing caveolin-1 expression; and could uncouple eNOS activity by inhibiting the association of $\mathrm{HSP} 90$ with eNOS. All these factors contributed to decreased NO production and increased $\mathrm{O}_{2} \bullet^{-}$generation, which lead to impaired vasodilation and hemodynamic instability. VEGF, Vascular endothelial growth factor; $n M P s$, MPs from healthy subjects; preoMPs, MPs from VHD patients before surgery; $12 \mathrm{~h}$ postoMPs, MPs from VHD patients at 12 hours after cardiac surgery; $72 h$ postoMPs, MPs from VHD patients at 72 hours after cardiac surgery; $R L U$, relative light units; $L-N A M E$, NG-nitro-L-arginine methyl ester; $C P B$, cardiopulmonary bypass; $M P$, microparticle; $H S P-90$, heat shock protein 90; eNOS, endothelial nitric oxide synthase; $P$-Akt, phosphorylation of Akt; $P$-PKC- $\beta I I$, phosphorylation of PKC- $\beta \mathrm{II}$; $P$-p70S6K, phosphorylation of p70S6K; $S$, serine; $N O$, nitric oxide; $\mathrm{O}_{2} \bullet^{-}$, superoxide anion.

site is very important for endothelial function. ${ }^{10,21}$ Protein kinase Akt can activate eNOS by phosphorylating eNOS on serine 1179 (1177), which leads to nitric oxide production. $^{22,23}$ Caveolin-1 is widely expressed in endothelial cells and can directly interact with eNOS, which can negatively affect eNOS activity and result in reduced generation of nitric oxide. ${ }^{24}$

Activation of PKC- $\beta$ II inhibits Akt-dependent eNOS from activating phosphorylation at Ser1177, and promotes phosphorylation of eNOS at Thr495, which leads to decreased eNOS activity. ${ }^{25,26}$ Activation of p70S6K, the downstream target of PKC- $\beta$ II, was considered to be activating phosphorylation of eNOS at the inhibitory site Thr495. ${ }^{26}$ Taken together, MPs from VHD patients could inhibit eNOS activation and reduce nitric oxide generation via inhibition of the Akt-eNOS pathway, the activation of the PKC- $\beta$ II $/$ p70S6K-eNOS pathway, and increased expression of caveolin-1. These findings highlight the harmfulness of MPs in VHD patients, especially after cardiac surgery, for negative regulation of eNOS.

We and other researchers demonstrated that heat shock protein 90 played an important role in coupling eNOS, to maintain the balance of eNOS-dependent nitric oxide and superoxide anion production. Inhibition of heat shock protein 90 associated with eNOS would promote superoxide anion generation. ${ }^{27}$ In the present study, we found that MPs from VHD patients, especially after surgery, decreased nitric oxide production and increased superoxide 
anion generation, which was inhibited by L-NAME. MPs from VHD patients, especially from after surgery, strongly inhibited the association of heat shock protein 90 with eNOS, indicating that MPs from VHD can uncouple eNOS to generate superoxide anion instead of nitric oxide, thereby leading to endothelial dysfunction. The findings suggest that both preoperative and postoperative MPs from VHD patients can impair endothelium-dependent vascular function by inhibiting eNOS activity.

\section{Limitations}

Currently, no reliable method is available to separate MPs of different originals for the blood samples, making it impossible to distinguish the effects of each MP subtype. The MPs that are generated in vitro cannot completely reproduce the function of in vivo MPs; thus, only the effect of all MPs combined was examined.

\section{CONCLUSIONS}

Our data show that MPs from VHD patients can impair endothelium-dependent vasodilation by decreasing nitric oxide production and increasing superoxide anion generation, which was caused by uncoupling of eNOS and inhibition of its activity. Given that this effect was more prominent during the perioperative period after cardiac surgery, it could induce hemodynamic abnormality (Figure 4, C). Our findings suggest that MPs are potential therapeutic targets for maintaining vascular function after cardiac surgery.

\section{Conflict of Interest Statement}

Authors have nothing to disclose with regard to commercial support.

The authors thank Professor Li Ling from the Department of Medical Statistics and Epidemiology of the School of Public Health, Sun Yat-sen University, for statistical analysis assistance, and professor Dong-Hong Liu from the Department of Ultrasound, the patients and staff at the First Affiliated Hospital, Sun Yat-sen University, for their assistance throughout this study.

\section{References}

1. Mallat Z, Benamer H, Hugel B, Benessiano J, Steg PG, Freyssinet JM, et al. Elevated levels of shed membrane microparticles with procoagulant potential in the peripheral circulating blood of patients with acute coronary syndromes. Circulation. 2000;101:841-3.

2. Montoro-Garcia S, Shantsila E, Tapp LD, López-Cuenca A, Romero AI, Hernández-Romero D, et al. Small-size circulating microparticles in acute coronary syndromes: relevance to fibrinolytic status, reparative markers and outcomes. Atherosclerosis. 2013;227:313-22.

3. Ci HB, Ou ZJ, Chang FJ, Liu DH, He GW, Xu Z, et al. Endothelial microparticles increase in mitral valve disease and impair mitral valve endothelial function. Am J Physiol Endocrinol Metab. 2013;304:E695-702.

4. Boilard E, Nigrovic PA, Larabee K, Watts GF, Coblyn JS, Weinblatt ME, et al. Platelets amplify inflammation in arthritis via collagen-dependent microparticle production. Science. 2010;327:580-3.

5. Martin S, Tesse A, Hugel B, Martínez MC, Morel O, Freyssinet JM, et al. Shed membrane particles from $\mathrm{T}$ lymphocytes impair endothelial function and regulate endothelial protein expression. Circulation. 2004;109:1653-9.
6. He GW. Endothelial function related to vascular tone in cardiac surgery. Heart Lung Circ. 2005;14:13-8.

7. Preventza O, Garcia A, Cooley DA, Tuluca A, Simpson KH, Bakaeen FG, et al. Reoperations on the total aortic arch in 119 patients: short- and mid-term outcomes, focusing on composite adverse outcomes and survival analysis. J Thorac Cardiovasc Surg. 2014;148:2967-72.

8. Chang FJ, Yuan HY, Hu XX, Ou ZJ, Fu L, Lin ZB, et al. High density lipoprotein from patients with valvular heart disease uncouples endothelial nitric oxide synthase. J Mol Cell Cardiol. 2014;74:209-19.

9. Boulanger CM, Scoazec A, Ebrahimian T, Henry P, Mathieu E, Tedgui A, et al. Circulating microparticles from patients with myocardial infarction cause endothelial dysfunction. Circulation. 2001;104:2649-52.

10. Ou ZJ, Chang FJ, Luo D, Liao XL, Wang ZP, Zhang X, et al. Endotheliumderived microparticles inhibit angiogenesis in the heart and enhance the inhibitory effects of hypercholesterolemia on angiogenesis. Am J Physiol Endocrinol Metab. 2011;300:E661-8.

11. Xuan C, Chang FJ, Liu XC, Bai XY, Liao XL, He GW, et al. Endothelial nitric oxide synthase enhancer for protection of endothelial function from asymmetric dimethylarginine-induced injury in human internal thoracic artery. J Thorac Cardiovasc Surg. 2012;144:697-703.

12. Owens AP III, Mackman N. Microparticles in hemostasis and thrombosis. Circ Res. 2011;108:1284-97.

13. Densmore JC, Signorino PR, Ou J, Hatoum OA, Rowe JJ, Shi Y, et al. Endothe lium-derived microparticles induce endothelial dysfunction and acute lung injury. Shock. 2006;26:464-71.

14. Brodsky SV, Zhang F, Nasjletti A, Goligorsky MS. Endothelium-derived microparticles impair endothelial function in vitro. Am J Physiol Heart Circ Physiol. 2004;286:H1910-5.

15. Levy JH, Tanaka KA. Inflammatory response to cardiopulmonary bypass. Ann Thorac Surg. 2003;75:S715-20.

16. Schmartz D, Tabardel Y, Preiser JC, Barvais L, d'Hollander A, Duchateau J, et al. Does aprotinin influence the inflammatory response to cardiopulmonary bypass in patients? J Thorac Cardiovasc Surg. 2003;125:184-90.

17. Almansob MA, Xu B, Zhou L, Hu XX, Chen W, Chang FJ, et al. Simvastatin reduces myocardial injury undergoing noncoronary artery cardiac surgery: a randomized controlled trial. Arterioscler Thromb Vasc Biol. 2012; 32:2304-13.

18. Biro E, van den Goor JM, de Mol BA, Schaap MC, Ko LY, Sturk A, et al. Complement activation on the surface of cell-derived microparticles during cardiac surgery with cardiopulmonary bypass-is retransfusion of pericardial blood harmful? Perfusion. 2011;26:21-9.

19. van den Goor JM, Nieuwland R, van Oeveren W, Rutten PM, Tijssen JG, Hau CM, et al. Cell Saver device efficiently removes cell-derived microparticles during cardiac surgery. J Thorac Cardiovasc Surg. 2007;134:798-9.

20. Landmesser U, Hornig B, Drexler H. Endothelial function: a critical determinant in atherosclerosis? Circulation. 2004;109:II27-33.

21. Pan X, Zheng Z, Hu S, Li S, Wei Y, Zhang Y, et al. Mechanisms of pulmonary hypertension related to ventricular septal defect in congenital heart disease. Ann Thorac Surg. 2011;92:2215-20.

22. Klinkner DB, Densmore JC, Kaul S, Noll L, Lim HJ, Weihrauch D, et al. Endothelium-derived microparticles inhibit human cardiac valve endothelial cell function. Shock. 2006;25:575-80.

23. Shi F, Wang YC, Zhao TZ, Zhang S, Du TY, Yang CB, et al. Effects of simulated microgravity on human umbilical vein endothelial cell angiogenesis and role of the PI3K-Akt-eNOS signal pathway. PloS One. 2012;7:e40365.

24. Mineo C, Shaul PW. Regulation of eNOS in caveolae. Adv Exp Med Biol. 2012; 729:51-62.

25. Besler C, Heinrich K, Rohrer L, Doerries C, Riwanto M, Shih DM, et al. Mechanisms underlying adverse effects of HDL on eNOS-activating pathways in patients with coronary artery disease. J Clin Invest. 2011;121: 2693-708.

26. Adams V, Besler C, Fischer T, Riwanto M, Noack F, Höllriegel R, et al. Exercise training in patients with chronic heart failure promotes restoration of highdensity lipoprotein functional properties. Circ Res. 2013;113:1345-55.

27. Ou J, Fontana JT, Ou Z, Jones DW, Ackerman AW, Oldham KT, et al. Heat shock protein 90 and tyrosine kinase regulate eNOS NO generation but not NO bioactivity. Am J Physiol Heart Circ Physiol. 2004;286:H561-9.

Key Words: Microparticles, valvular heart disease, cardiac surgery, vasodilation, endothelial nitric oxide synthase 